\title{
Relationship Between Metronidazole and Co-Trimoxazole on Eradication of Entamoeba Histolytica
}

\author{
${ }^{1}$ Mohammed Aladwan, ${ }^{2}$ Mohammed Ali Abu-Rumman, ${ }^{3}$ Yazun Bashir Jarrar, ${ }^{1}$ Eman Khreisat, \\ ${ }^{1}$ Amal Farhan Khreisat, ${ }^{1}$ Sameer Mahmoud Alshiab, ${ }^{1}$ Nadia Al Smadi, ${ }^{1}$ Ahamad Al-Ali FBMS, \\ ${ }^{1}$ Fatima Mohammad Aburumman, ${ }^{1}$ Ghadah Al Abdallat and ${ }^{1}$ Ftimeh Naemat \\ ${ }^{1}$ Department of Emergency and Pediatric Emergency Clinic of Queen Alia Military Hospital, Jordan \\ ${ }^{2}$ Department of Nurse Registration, Albalqu Univercity, Jordan \\ ${ }^{3}$ Department of Pharmacology College of Medicine, Inje University, South Korea
}

\begin{abstract}
To present the efficacy and safety of Metronidazole and co-trimoxazole in the treatment of diarrhea caused by Entamoeba histolytica, cyst and trophozyed in patients presented to the emergency department and emergency pediatric clinic in Queen Alia Hospital (Jordan). A strategy for diagnosis Entamoeba histolytica was evaluated by studying 181 patients aged 2-50 years. Between the 1st of May 2009 and the beginning of May 2010, 181 patients with intestinal amoebiasis were recruited for this study from the Emergency Department and emergency Pediatric Clinic at Queen Alia Military Hospital. After doing stool analysis for every patient, Metronidazole $500 \mathrm{mg}$ three times was given to the adult group and $40 \mathrm{mg} \mathrm{kg} \mathrm{day}^{-1}$ three divided doses for the pediatric group for 10 days and two tablets twice daily for adults and $5 \mathrm{~mL}$ twice daily for children for ten days of co-trimaxazole, with follow up stool analysis after one week of treatment. Out of 181 patients (81) patients were adult's age (14-50 years) and (100 patients) were children aged (2-14 years). About $5.5 \%$ of the patient showed the amoebic trophozoite in their stool while the remaining showed only the amoebic cysts. Over all metronidazole and co-trimoxazole produced a clinical response rate of $95 \%$ in both adults and children group after 10 days course of Metronidazole. Our findings demonstrated the efficacy of 10 day of course Metronidazole and co-trimoxazole in eradication intestinal amoebiasis for which the causative organism is identified by simple stool examination.
\end{abstract}

Keywords: Entamoeba Histolytica Cyst, Metronidazole (Dumozol), Septrin (Co-Trimoxazole), Entamoeba Histolytica Trophozoite

\section{INTRODUCTION}

Enteric protozoan infections are among the most common infections in human's worldwide and significant cause of morbidity and mortality.

Symptomatic patients typically present with diarrhea and abdominal symptoms including cramps, pain and bloating. Although a number of protozoa may cause these symptoms, but Giardia intestinal and $E$. histolytica are the most commonly reported. Amoebiasis (Amoebic dysentery) is an infectious disease caused by protozoan parasite called Entamoeba histolytica .It causes colitis characterized by painful passage of bloody mucoid stool (Schuster and RamirezAvila, 2008; Lourenssen et al., 2010).

Entamoeba histolytica is protozoan parasite, found world wide and $12 \%$ of the world's population are estimated to be infected. The prevalence of infection varies between $1 \%$ in developed countries to $50-80 \%$ in tropical countries where transmission of E. histolytica cyst from human by untreated drinking water is common. Ingestion of food and drink, contaminated Hospital, Jordan 
with $E$. histolytica cyst from human faeces and direct faecal oral contract are the commonest means of infection (Kreidl et al., 1999). Cyst carries are the main reservoir of infection. Cysts may remain viable for three months but may be destroyed by hyper chlorination or iodination of the drinking water. About $10 \%$ of patients infected have clinical symptoms, $80-$ $98 \%$ with amoebic colitis, 2-0\% with extra-intestinal disease (liver abscess). The clinical picture of amoebic colitis is characterized by abdominal pain, cramps, generalized, weakness, tenesmus and passage of blood and mucus in the stool (Anand et al., 1997).

Chronic abdominal pain and frequent bowel disturbances are common symptoms experienced by more than $15 \%$ of apparently healthy people. In areas endemic for E. histolytica infection, these symptoms are often diagnosed as non-dysenteric intestinal amoebiasis and irritable bowel syndrome is not distinct (Schuster, 1993).

Metronidazole is the most popular drug of choice in treatments of E. histolytica infections which is given either orally or intravenously especially in severs cases. It has a low toxicity and it is effective against both intestinal and extra-intestinal amoebiasis (Tanyuksel et al., 2005).

Ingestion of the quadrinucleate cyst of E. histoliytica from fecally contaminated food or water initiates infection. Infection with $E$. histolytica may be asymptomatic or may cause dysentery or extra intestinal disease (8).

\section{MATERIALS AND METHODS}

The study protocol was designed with reference to guidelines proposed for the evaluation of Metronidazole and Septrin (co-trimoxazole) for the treatment of amoebic colitis.

Between the 1st of May 2009 and the beginning of May 2010, (181) patients aged (2-50 years) with intestinal amoebiasis were recruited for this study, from the Emergency department and Pediatric emergency Clinic of Queen Alia Military Hospital, Patients with gastrointestinteritis due to other causes like Rota virus Giardia, Shigella were excluded from our study.

After doing stool analysis by inspection and microscopic examination for every patient, Metronidazole $500 \mathrm{mg}$ three times daily was given to the adult group and $40 \mathrm{mg} \mathrm{kg} \mathrm{day}{ }^{-1}$ in three divided doses for the pediatric group for 10 days, in addition to nutritional and fluid support. About $20 \%$ of children required admission for dehydration and $5 \%$ of adults required admission because of constitutional symptoms life fever, hypotension and association with chronic illness like Diabetes mellitus.

Follow up of the study group was done by taking history, physical examination and stool analysis after one week and repeated after 2 weeks of treatment.

\section{RESULTS}

Faecal samples of (181) patients adults age (15-50 years) and children age (2-14 years) were screened for identifying the causes of the disease. The samples were analysed by microscopic examination and found that $(82.8 \%)$ (males and females) have liquid stool and (7.4\%) have semisolid stool.

The median duration of diarrhea at the time of enrollment was 5 days $(86 \%)$ of the patients reported 5 10 motion per day but the reminder reported more than 10 motions per day. (80\%) of the patients reported a complaints of abdominal pain cramps and distension. $(11.1 \%)$ showed blood in their stool. (5.5\%) showed the amoebic trophozoite while the remaining patients showed cysts of Entamoeba histolytica. Over all Metronidazole produced a response rate of $95 \%$ after a 10 day course of Metronidazole.

The duration of diarrhea in adult males and females was $(3-5$ days) in $(79.6 \%, 76.9 \%)$ respectively which was less than that of children ( $85.8 \%$ males, $83.3 \%$ females) also the duration of diarrhea was more in males than females in both adults and children as seen in the table.

\section{DISCUSSION}

In this study, our findings demonstrated the efficacy of 10 day course of Metronidazole in resolving diarrhea for which the causative organism was identified by microscopic examination of stool samples to be Entamoeba histolytica, so Metronidazole which is an amoebicidal and bactericidal agent with low toxicity is the drug of choice to treat acute amoebic colitis and it is found that Metronidazole is effective against amoebic liver abscess (McAuley et al., 1992; Gonzales et al., 2009), although surgical aspiration may be needed sometime, but it is demonstrated that use of another agent in addition to Metronidazole that is active against cysts is needed in some cases like Septrin which has efficacy rate of more than $90 \%$ in eradication of the amoebic cysts (McAuley et al., 1992), but the use of this agent is limited because of its adverse effects (Gastrointestinal and neurological side effects which were produced in $9 \%$ of the patients given the treatment after 14 years experience with the use of Septrin. 
Mohammed Aladwan et al. / American Journal of Microbiology 3 (2): 31-33, 2012

Table 1. Age and sex distributionand results of stool examination according to study group

\begin{tabular}{|c|c|c|c|c|c|c|c|c|}
\hline \multirow[b]{2}{*}{ Age (years) } & \multirow[b]{2}{*}{$\begin{array}{l}\text { Sex (no. of } \\
\text { Patient) }\end{array}$} & \multicolumn{2}{|c|}{ Duration of diarrhea } & \multicolumn{2}{|c|}{ Stool frequency } & \multicolumn{3}{|c|}{ Microscopic examination } \\
\hline & & $\begin{array}{l}3-5 \\
\text { days }(\%)\end{array}$ & $\begin{array}{l}\text { More than } \\
5 \text { days (\%) }\end{array}$ & $\begin{array}{l}\text { Liquid } \\
\text { stool (\%) }\end{array}$ & $\begin{array}{l}\text { Soft } \\
\text { stool (\%) }\end{array}$ & $\begin{array}{l}\text { Occult } \\
\text { blood (\%) }\end{array}$ & $\begin{array}{l}\text { Mucus } \\
(\%)\end{array}$ & $\begin{array}{l}\text { Pus } \\
(\%)\end{array}$ \\
\hline \multirow{2}{*}{ Adults (15-50) } & Males (55) & 79.6 & 20.4 & 85.5 & 14.5 & 7.0 & 90 & 100 \\
\hline & Females (26) & 76.4 & 23.1 & 88.5 & 11.5 & 7.7 & 100 & 100 \\
\hline \multirow[t]{2}{*}{ Children (2-14) } & Males (64) & 85.9 & 14.1 & 93.7 & 6.3 & 15.6 & 100 & 100 \\
\hline & Females (36) & 83.3 & 16.7 & 97.2 & 2.8 & 13.9 & 100 & 100 \\
\hline
\end{tabular}

The clinical syndrome of non-dysenteric intestinal amoebiasis or chronic amoebiasis has been a controversial entity for sometime. Although accepted by $\mathrm{WHO}$, the definitive diagnosis of non-dysenteric intestinal amoebiasis has been thrown into doubt by new methods of investigation. In this study we compared patients who had dysenteric intestinal amoebiasis with a few who had non-dysenteric intestinal amoebiasis (who had soft stool) as seen in Table 1, which showed similar response to the study medication (Lynn and Friedman, 1993; Verma et al., 2010).

Clinical and parasitological response rates for the nitazoxamide treatment group were similar to those reported for open-label studies of Augminten with Metronidazole for amoebiasis and giardiasis (Goessling and Chung, 2002).

The study emphasizes the importance of 10Day course of Metronidazole and septrin in treatment of invasive amoebiasis and considering its use in asymptomatic cyst passers who didn't respond to Metronidazole and become cyst carriers who needed another luminal agent to eradicate the cysts. Controversy remains over which agent to use and this will require further comparative trials.

\section{CONCLUSION}

It is illustrated in this study that Metronidazole and septrin can be very effective in treating amoebic colitis and abdominal pain, especially if it is given for two week period, although we have few patients, who became cyst carriers.

The authors end by saying that another intraluminal agent such as Septrin should be given for treating asymptomatic patient who are passing Entamoeba histolytica cysts.

\section{REFERENCES}

Anand, A.C., P.S. Reddy, G.S. Saiprasad and S.K. Kher, 1997. Does non-dysenteric intestinal amoebiasis exist? Lancet, 349: 89-92. PMID: 8996420
Goessling, W. and R.T. Chung, 2002. Amebic liver abscess. Curr. Treat Options Gastroenterol., 5: 443449. PMID: 12408781

Gonzales, M.L., L.F. Dans and E.G. Martinez, 2009. Antiamoebic drugs for treating amoebic colitis. Cochrane Database Syst. Rev. PMID: 19370624

Kreidl, P., P. Imnadze, L. Baidoshvili and D. Greco, 1999. Investigation of an outbreak of amoebiasis in Georgia. Eur. Surveil., 4: 103-104. PMID: 12631887

Lourenssen, S., E.R. Houpt, K. Chadee and M.G. Blennerhassett, 2010. Entamoeba histolytica infection and secreted proteins proteolytically damage enteric neurons. Infect. Immunol., 78: 53325340. DOI: 10.1128/IAI.00699-10

Lynn, R.B. and L.S. Friedman, 1993. Irritable bowel syndrome. N Engl. J. Med., 329: 1940-1945. DOI: 10.1056/NEJM199312233292608

McAuley, J.B., B.L. Herwaldt, S.L. Stokes, J.A. Becher and J.M. Roberts et al., 1992. Diloxanide furoate for treating asymptomatic Entamoeba histolytica cyst passers: 14 years' experience in the United States. Clin. Infect. Dis., 15: 464-468. PMID: 1520794

Schuster, F.L. and L. Ramirez-Avila, 2008. Current world status of Balantidium coli. Clin. Microbiol. Rev., 21: 626-638.

Schuster, M.M., 1993. Irritable bowel syndrome: New perspectives on management. Cleve Clin. J. Med., 60: 270-271. PMID: 8339451

Tanyuksel, M., H. Yilmaz, M. Ulukanligil, E. Araz and M. Cicek et al., 2005. Comparison of two methods (microscopy and enzyme-linked immunosorbent assay) for the diagnosis of amebiasis. Exp. Parasitol., 110: 322-326. PMID: 15955332

Verma, G. K., N. L. Sharma, V. Shanker, V.K. Mahajan and R. Kaushik et al., 2010. Amoebiasis cutis: Clinical suspicion is the key to early diagnosis. Aus. J. Dermatol., 51: 52-55. PMID: 20148845 